Method. EEG data were recorded simultaneously with BOLD data measured with $3 \mathrm{~T}$ functional magnetic resonance imaging (fMRI), whilst participants performed an n-back working memory task. We included seventy-eight participants - 32 patients with schizophrenia, 16 with bipolar disorder and 30 healthy controls. Beta bursts were identified in the EEG data using a thresholding method and burst timings were used as markers in an event-related fMRI design convolved with a conventional haemodynamic response function. A region of interest analysis compared beta-event-related BOLD activity between patients and controls.

Result. Beta bursts phasically activated brain regions implicated in coding task-relevant content (specifically, regions involved in the phonological representation of letter stimuli, as well as areas representing motor responses). Further, bursts were associated with suppression of tonically-active regions. In the EEG, PMBR was greater in controls than patients, and, in patients, PMBR size was positively correlated with Global Assessment of Functioning scores, and negatively correlated with persisting symptoms of disorganisation and performance on a digit symbol substition test. Despite this, patients showed greater, more extensive, burst-related BOLD activation than controls.

Conclusion. Our findings are consistent with a recent model in which beta bursts serve to reactivate latently-maintained, task-relevant, sensorimotor information. The increased BOLD response associated with bursts in patients, despite reduced PMBR, could reflect inefficiency of burst-mediated cortical synchrony, or it may suggest that the sensorimotor information reactivated by beta bursts is less precisely specified in psychosis. We propose that dysfunction of the mechanisms by which beta bursts reactivate task-relevant content can manifest as disorganisation and working memory deficits, and may contribute to persisting symptoms and impairment in psychosis.

\section{Evaluating service user \& carer experience of videoconferencing software during COVID-19 pandemic}

Joseph MacDonnell

Kendray Hospital

doi: 10.1192/bjo.2021.152

Aims. To evaluate service user and carer experience of use of videoconferencing software (Microsoft Teams) during MDT meetings.

To identify specific areas for improvement

To make changes based on these recommendations

Method. 2 surveys were distributed to inpatients and their carers on a functional Older Adults inpatient ward $(\mathrm{n}=21)$, including quantitative and qualitiative questions. The results from these were compiled, and on review, mutliple recommendations for improvement were made.

Result. 90\% of service users find it helpful to have family present over videoconferencing software during their MDT meetings, and $91 \%$ of carers feel involved and able to contribute when they do join in this way

$81 \%$ of carers have the technology available at home to use such software, but only 55\% of them feel confident using it. $73 \%$ need more information on its use.

$60 \%$ of carers referenced poor staff skills with software as a barrier to its use, and $60 \%$ referenced poor organisation of meetings

2 service users raised issue with the size of a small laptop screen not allowing them to see who was actually present over MS Teams, although none were concerned with issues around confidentiality and the use of such software
Several service users, carers and members of community teams identified poor sound quality as an issue, both when joining over the software, and when present in the room.

Conclusion. Widespread use of videoconferencing software such as MS Teams is likely to continue beyond the end of the COVID-19 pandemic. Through discussion with the ward team, the IT department, the training department, and the local council, multiple changes were made to the service, as below. These form a recommended list of areas for improvement in other services.

Availability of videoconferencing equipment (in addition to laptop)

Dedicated videoconferencing microphone/speaker to improve sound quality

Display screen

Webcam

Organisation of meetings

Designating a chairperson to admit and introduce all participants

Designating a meeting organiser to invite all necessary participants

Staff skills

Local audit of staff familiarity with software

Introduction of mandatory training for staff on use of software Carer skills \& access to equipment

Information and support available from well-trained staff

Liaison with other organisations including council and third sector about availablity of equipment loans and training for carers

Using quality improvement to standardise and enhance the use of the national early warning score (NEWS) in an old age psychiatry inpatient setting

Fraser MacNicoll ${ }^{1 \star}$, Mong Sun Tung ${ }^{2}$ and Brion McGowan ${ }^{2}$

${ }^{1}$ Psychiatry of Old Age, NHS Lothian and ${ }^{2}$ NHS Lothian

${ }^{*}$ Corresponding author.

doi: 10.1192/bjo.2021.153

Aims. Within an inpatient old-age psychiatry setting, there is an increased risk of acute physical deterioration secondary to age, comorbidities and reduced physiological reserve. Numerous recent clinical incidents highlighted late recognition of physical deterioration within this population. We assessed the use of the NEWS, a system for scoring physiological measurements, in an old-age psychiatry ward and subsequently attempted to improve performance of obtaining physical health observations in this cohort of patients.

Method. Retrospective pre- and post- quality improvement study in a twenty bed Old Age Psychiatry Ward in East Lothian Community Hospital, Haddington, Scotland. Data were collected from 12th October - 16th November, 2020 (pre- period) and from 16th November 2020 to 15th February, 2021 (post- period). The primary process measure was ensuring all patients had at least one full set of physical observations at least once a week, or more frequent as deemed clinically appropriate. Secondary measures included ensuring NEWS scores were accurately calculated and improved documentation. This was tracked using a run chart. Improvement activities focused on increased awareness, effective training, key stakeholder buy-in and reviewing trust policy.

Result. The percentage of NEWS documented for all patients at least once a week improved from a mean of $28.7 \%(31 / 108)$ in the 6 weeks prior to intervention, to a mean of $71.4 \%(125 / 175)$ in the following 13 weeks. The minimum required physical 